\title{
A COOPERATIVE BAIT DETECTION APPROACH USING POWER EFFICIENT DYNAMIC SOURCE ROUTING FOR DETECTING MALICIOUS NODES IN MANETS
}

\author{
Bharath $k^{1}$, Salonee Mishra ${ }^{2}$ \\ ${ }^{1}$ Student, Computer Network and Engineering, TJIT, Karnataka, India \\ ${ }^{2}$ Associate professor, Computer Science and Engineering, TJIT, Karnataka, India
}

\begin{abstract}
In mobile adhoc system beginning prerequisite for setting up correspondences among systems is that every one of the hubs ought to participate with each other. Because of the malicious hubs present in the system the hubs may upset the directing procedure. In this paper we are showing another methodology i.e. Power efficient dynamic source routing process [PEDSR], fulfilling less power utilization from the perspectives of hubs and system. To accomplish our objective, initially, we concentrated on DSR convention utilizing execution and force mindful measurements. Changes are done on the Dynamic Source Routing (DSR) convention by thinking about the results of the pre-reproduction, the current component of DSR for usage of the outline, and the past examination works done on DSR directing convention by numerous specialists. Therefore, we considered the execution assessment of our recommendation. The reenactment results demonstrate that the force mindful steering convention, PEDSR outflanks the first DSR convention in larger part of the situations and assessment measurements. Also, helpful lure identification approach Implements reverse following method for identifying of malignant hubs present in the system. It beats the upsides of both proactive and responsive plans furthermore utilizing reverse following procedure the pernicious hubs Information is found and kept operating at a profit gap list. The force effective element source directing is utilized i.e. the hubs that have more vitality that hubs is utilized for directing procedure. Recognizing of dark opening and dim gap assault are done in view of helpful trap discovery plan.
\end{abstract}

Keywords: Power Efficient Dynamic Source Routing, Cooperative Bait Detection Scheme, Collaborative Black Hole Attack, Gray Hole Attack, Detection Mechanism.

\section{INTRODUCTION}

Because of the boundless of cell phones Mobile adhoc networks [MANETS] has been utilized as a part of assortment of utilizations, for example, crisis operations, for example, military applications and so on... In MANETs every one of the hubs ought to participate with each other furthermore while getting information the hubs ought to collaborate with each other to forward the information bundles here every hub goes about as a switch. Amid the transmission of parcels in the system the malignant hubs may upset the steering process in system operations.

In versatile adhoc systems numerous analysts have been centered on different steering process furthermore different plans of recognition instruments. For directing procedure Power efficient dynamic source routing procedure is utilized where Dynamic source routing includes course revelation and course support where the source hub sends the course demand to all the neighbor hubs if the middle of the road hubs have the directing data to the destination hub the it will give course answer to the source hub that it has the most limited way to the destination and here force effective element source steering includes less power utilization for hubs in the system. PEDSR is gotten from DSR convention. PEDSR utilizes transmission power control approach which can be accomplished with the assistance of topology control of a MANET. The extent over which the sign is intelligently gotten by the recipient is controlled by the transmission power. PEDSR convention in view of transmission force finds the beat course that minimizes the aggregate transmission power between a source and destination.

\section{RELATED WORK}

Without a settled foundation, impromptu systems need to depend on compact, constrained force sources. Besides, hub in a specially appointed system needs to transfer messages for different hubs in the same system. The issue of force effectiveness in this way gets to be a standout amongst the most essential issues in specially appointed systems. Force can be devoured amid handling and correspondence. The force expended amid correspondence is more overwhelming than the force devoured amid handling. In this way, the correspondence framework ought to be force productive or power mindful by advancing the force utilization at various conditions of the correspondence. Power Aware Routing is extremely key in MANET.

Numerous scientists have explored the issue of discovery of noxious hubs however the vast majority of the identification components bargains just with the single location system. 
As a rule, location components that have been proposed so far can be assembled into two general classifications. Proactive getection plans are plans that need to continually identify or screen close-by hubs. In these plans, paying little heed to the presence of noxious hubs, the overhead of discovery is continually made, and the asset utilized for recognition is always squandered. In any case, one of the benefits of these sorts of plans is that it can help in counteracting or keeping away from an assault in its underlying stage. Responsive identification plans are those that trigger just when the destination hub distinguishes a critical drop in the parcel conveyance proportion.

Black hole and gay hole attacks are the two sorts of assailants that are available in the system. The black hole node i.e. the malevolent hubs send course demand to all the neighbor hubs saying that it has the briefest way to the destination and if any neighbor sends answer parcels then the pernicious hub will take every one of the bundles to the fake destination. In gray hole initially the malevolent hub is not perceived but rather at the later time when the parcels are exchanging that time pernicious hubs send course demand and takes every one of the bundles to the fake destination when neighbor hubs offers answer to noxious hubs.

\subsection{Motivation}

Power Aware directing strategy fundamentally goes for minimizing the force utilization of the system. We have experienced the writing study of different MANET steering convention. Every convention has critical significance of its own and additionally has a few confinements. In view of the confinement imperative of more power utilization in MANET we proposed another force proficient element source directing convention which utilizes jump by-bounce power control instrument to expand the lifetime time of both hub and additionally organize and lessens the aggregate force utilization in the system.

\subsection{Existing System}

Numerous specialists have concentrated on security of Mantes. The greater part of them manages counteractive action and discovery of malevolent hubs that make trouble in the steering process. In Black opening assaults as the noxious hubs get every one of the bundles that it has the briefest way to the destination yet parcels are taken to the fake destination. In Gray gap assaults initially it is not perceived but rather later it goes about as noxious hubs amid the transmission of parcels. In Dynamic source routing taking into account the course demand and course support directing procedure is finished.

\subsection{Proposed System}

Power efficient dynamic source routing is utilized for steering process as a part of location of noxious hubs in the system. Power productive element source steering utilizes less vitality utilization and agreeable trap identification approach utilizes reverse tracing procedure for recognizing of malicious hubs present in the system dispatching dim opening or community dark gap assaults. The helpful draw location plan defeats the benefits of proactive and reactive detection schemes.

The Cooperative detection approach Involves three stages they are Bait step, reverse tracing method, shifted to reactive defense method.

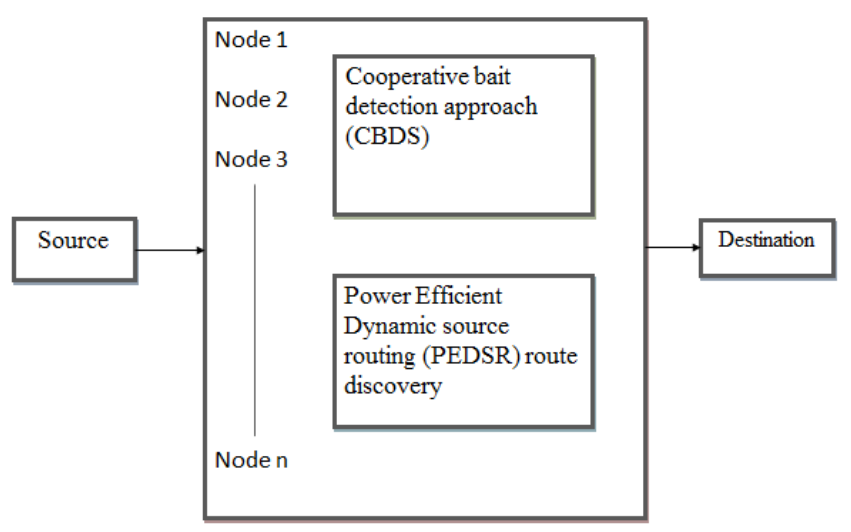

Fig -1: Architecture of proposed system

1] Bait step : In this identification approach Initial step is the trap step where as the source hub stochastically chooses the contiguous hubs of one jump neighbor and sends the RREQ' message to all the neighbor hubs and here nearby hubs would be changed if the hub is moved yet the goad would not stay unaltered. On the off chance that neighbor hub was the malignant hub then in the wake of sending RREQ' from the source then vindictive hubs would answer to the solicitation and acting up hubs are identified and kept operating at a profit opening rundown.

2] Reverse tracing technique : Here once the source hub sends the RREQ' message to neighbor hubs then the malignant hubs will answer back to the source hub then the source hub identifies getting rowdy hubs and location of the pernicious hubs is kept operating at a profit gap list. Presently the source hubs leave the malignant hubs and bundles are sent through another way.

3] Shifted to reactive defense: Once the getting out of hand hubs distinguished and kept in the operating at a profit gap list then the parcels are sent from source to destination. In the event that again bundle conveyance proportion is less at the destination on the other hand reverse following is connected to recognize the malignant hubs.

To acquire a force mindful steering convention we utilize power control approach i.e. Transmission power control approach. On the off chance that we contrast our proposed convention PEDSR and past works of specialists taking into account proposed model of DSR. In our proposed convention PEDSR the thought behind is that, a jump bybounce power control component is utilized to change the aggregate force utilization of the system. Hubs rich in influence are permitted to take an interest in directing procedure and over used hubs are evaded which may expend more influence and along these lines thusly the life time of 
the system is moved forward. In the event that there is a condition that the transmit force is settled and approach for every one of the hubs, then least bounce is the base force course. So with a specific end goal to increase most extreme force reserve funds, the base force directing convention ought to transmit the information parcel at force $\mathrm{t} P$ rather than the settled transmit power. This can be accomplished by applying dynamic transmit power control on the connection. Presently if dynamic transmit power control is utilized, the force expense of every connection can be figured utilizing t $\mathrm{P}$ and different parameters.

Despite the fact that a few conventions permit join measurements other than least jump, the current on-interest conventions don't offer any instruments to process and proliferate the parameters important to register the per bundle power cost. Thus the element transmit power control highlight can't be upheld by the current renditions of the oninterest conventions. Other than element transmit force is utilized for force preservation, it is utilized to use the system asset proficiently. That is by permitting a more prominent number of synchronous transmissions, the force control expand the aggregate system limit. In PEDSR, there is likewise a choice that if the highway tends to break sooner than the fancied period that breakage is recognized before and is evaded by including Least Power field.

The remaining battery force of a versatile hub is kept by the Minimum-Power field. At the point when a hub acknowledges a Route Request (RREQ) bundle from its neighboring hubs it looks at the Minimum-Power esteem in the parcel with its remaining force. On the off chance that the remaining forces is not as much as Minimum-Power, this force is doled out as the Minimum-Power. This procedure will proceed up to the destination. In the event that more than one RREQ from various courses is acknowledged by the destination, the course having the most elevated worth is chosen in the Minimum-Power field and after that Route Reply (RREP) bundle is sent to the source.
That implies a course is chosen by keeping away from the hub which is tending to terminate prior. Along these lines the courses which may break early are wiped out. A jump by-bounce control system is utilized to spare the remaining battery power in which the hubs that gets a Route-Request parcel at force Pr is transmitted with force Pt, then the new transmission power Pn for this accepting hub is ascertained such that this hub can Communicate with the sender hub by utilizing this base required force $\mathrm{n} \mathrm{P}$ utilizing the recipe

$P$ ntr $=P t-P r+P t h r+P m$

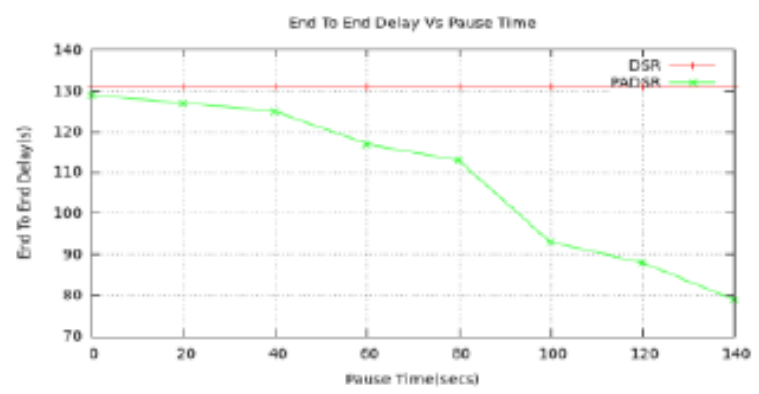

Fig -2: End to End delay for DSR and PEDSR

The operation of CBDS (Cooperative Bait Detection Scheme) demonstrates how the malevolent hubs are distinguished and kept operating at a profit opening rundown. The CBDS actualizes reverse following strategy to distinguish the getting rowdy hubs in the system. At first the source hub stochastically chooses an contiguous hub to draw course ask for It sends RREQ' message to all the neighbor hubs and if any of the hub send answer to the source then malevolent hubs Information is identified and hub data is kept operating at a profit gap list. This is the opposite following system connected to identify the getting out of hand hubs in the system.

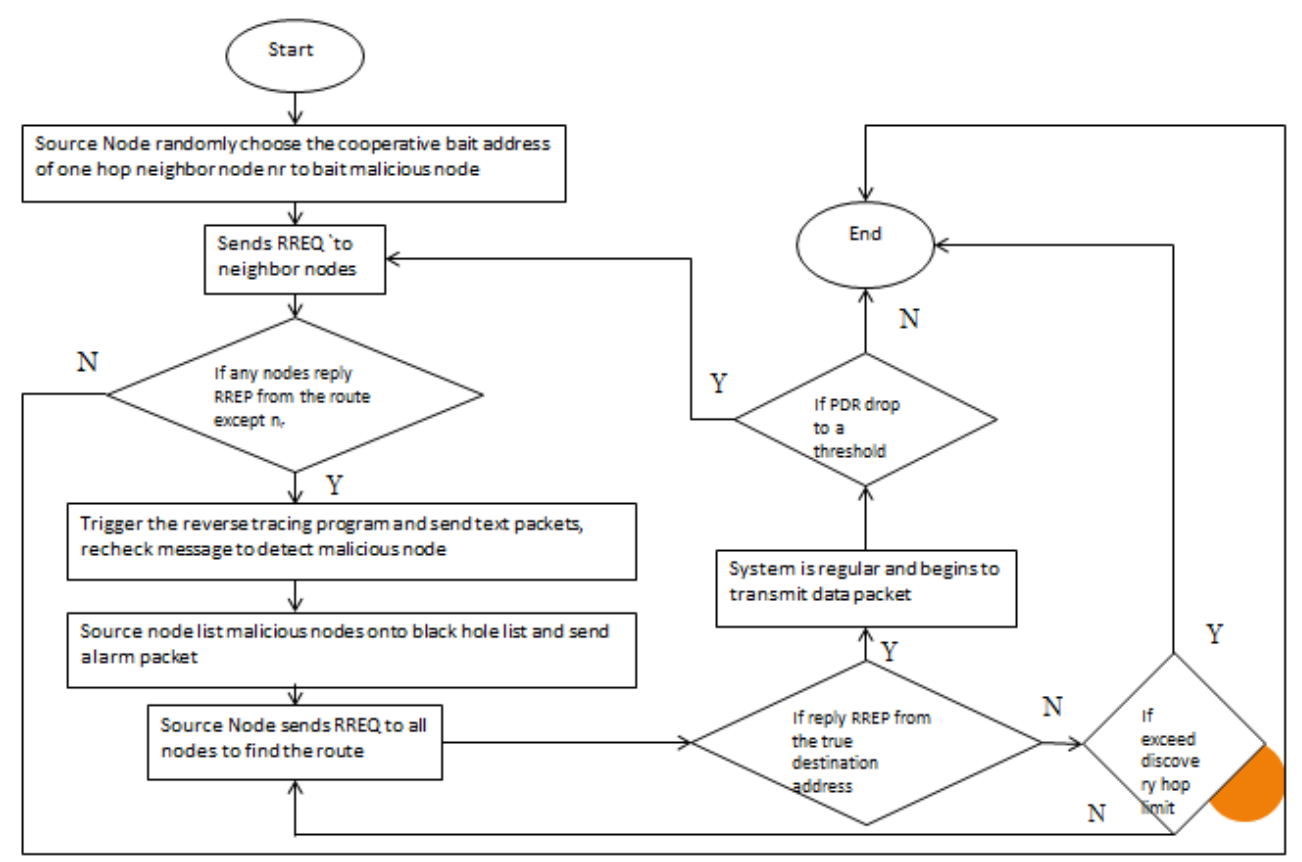

Fig -3: Operation of CBDS 
In the CBDS approach if the noxious hubs are available in the system then trigger the opposite following method to distinguish the getting out of hand hubs and again subsequent to identifying malignant hubs source hub sends course ask for parcel to every one of the hubs if course answer from the genuine destination then bundles are exchanged from source to the destination if not from genuine destination on the other hand reverse following is done to recognize the vindictive hubs.

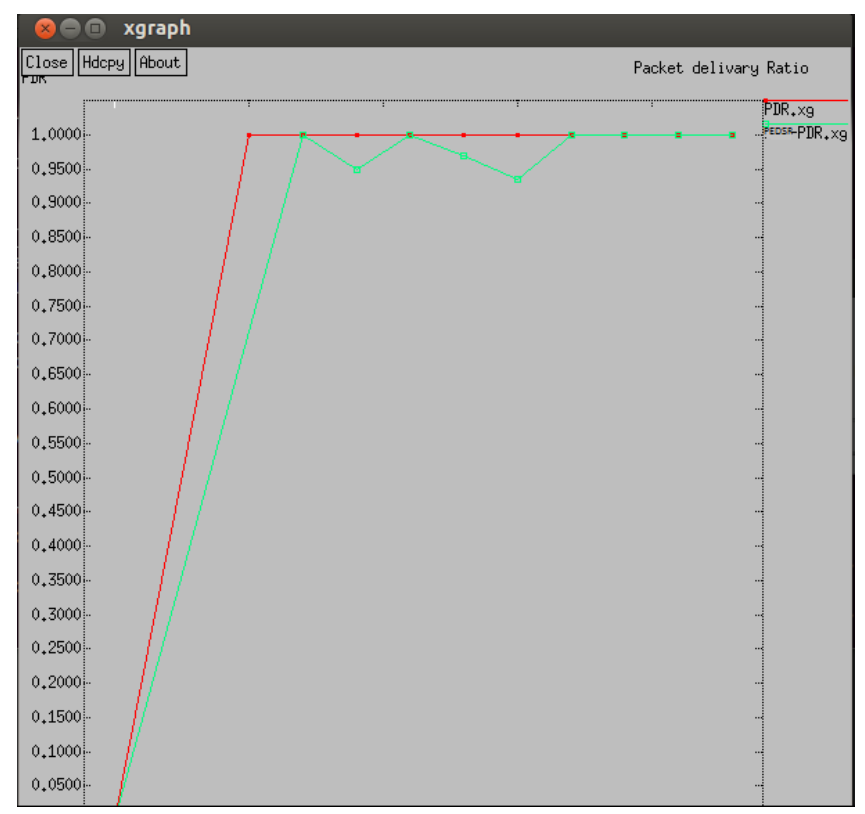

Fig -4: packet delivery ratio of PEDSR

The bundle conveyance proportion as appeared in the graph speaks to that pdr proportion is high more parcels are sent by utilizing power effective element source steering. In force proficient element source directing process more bundles can be conveyed when less power utilization is done in steering process.

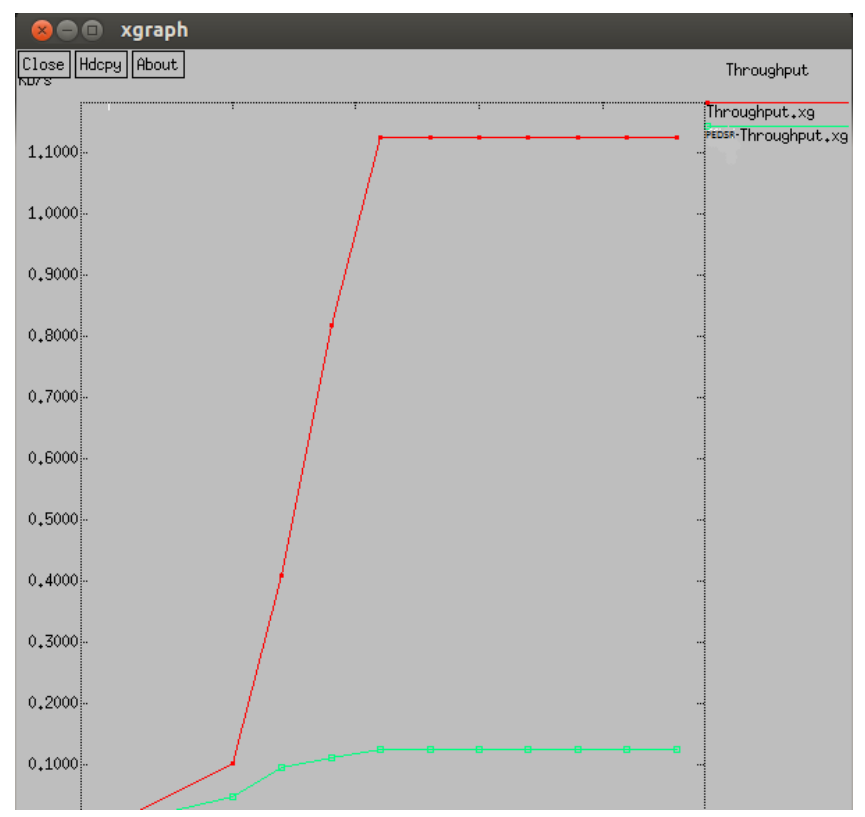

Fig -5: Throughput of PEDSR

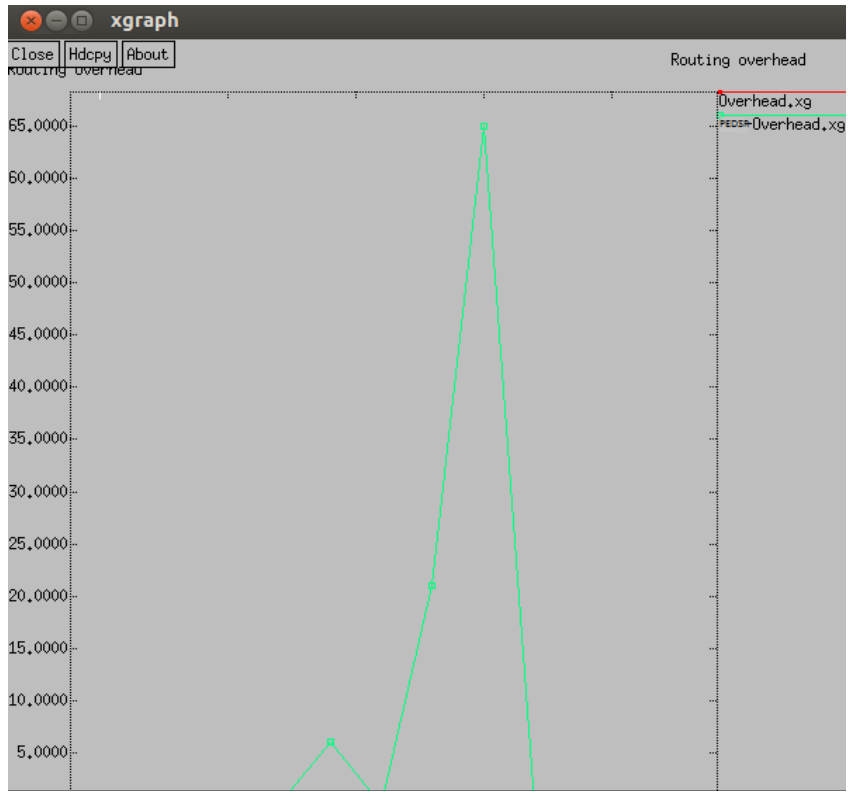

Fig -6: Routing overhead of PEDSR

In the above chart it speaks to the steering overhead of Power efficient dynamic source routing and portrays that by utilizing PEDSR less power utilization is utilized henceforth the best course is chosen for exchanging of bundles from source to the destination.

\section{CONCLUSIONS}

In this paper we have proposed another component (called power efficient dynamic source routing) for steering process where less vitality utilization is utilized so that the hubs comprises of more vitality just that hubs are chosen for steering procedure and agreeable draw location approach utilizes reverse tracing strategy for distinguishing of pernicious hubs in the system so that by utilizing reverse tracing method it beats the upsides of proactive and reactiveschemes furthermore black hole/gray holeattacks are additionally recognized in view of CBDS methodology.

\section{REFERENCES}

[1]. D. Johnson and D. Maltz, "Dynamic source routing in ad hoc wirelessnetworks," Mobile Comput., pp. 153-181, 1996.

[2]. I. Rubin, A. Behzad, R. Zhang, H. Luo, and E. Caballero, "TBONE: A mobile-backbone protocol for ad hoc wireless networks," in Proc. IEEE

Aerosp. Conf., 2002, vol. 6, pp. 2727-2740.

[3]. S. Marti, T. J. Giuli, K. Lai, and M. Baker, "Mitigating routing misbehavior in mobile ad hoc networks," in Proc. 6th Annu. Intl. Conf. MobiCom, 2000, pp. 255-265.

[4]. K. Vishnu and A. J Paul, "Detection and removal of cooperative black/gray hole attack in mobile ad hoc networks," Int. J. Comput. Appl.,vol. 1, no. 22, pp. 28-32, 2010.

[5]. K. Liu, D. Pramod, K. Varshney, and K. Balakrishnan, "An Acknowledgementbased approach for the detection of routing misbehavior in MANETs," IEEE Trans. Mobile Comput., vol. 6, no. 5, pp. 536-550,

May 2007. 